International Journal of Biology, Pharmacy

and Allied Seiences (IJBPAS)

'A Bridge Betusen Caboratory and QRendo'

WwW.iibpas.com

\title{
DEEP NEURAL NETWORK APPLICATION FOR MEDICAL IMAGE PROCESSING
}

\section{HEMALATHA.C ${ }^{*}$, NELSON KENNEDY BABU C ${ }^{2}$, RAJENDRA KANNAMMAL. G ${ }^{3}$ AND Dr. LAKSHMI B $\mathbf{N}^{4}$}

1: Assistant Professor in Computer Science and Engineering at Sathyabama Institute of Science and Technology, Jeppiaar Nagar, Rajiv Gandhi Salai, Chennai Tamilnadu, India

2: Professor in Computer Science and Engineering at Saveetha School of Engineering, Saveetha Institute of Medical and Technical Sciences, Chennai, India

3: Assistant Professor in Computer Science and Engineering at K. Ramakrishnan College of Technology, Samayapuram, Trichy, India

4: Department of Computer Science and Engineering, BMS Institute of Technology and Management, Bangalore, Karnataka, India

*Corresponding Author: Hemalatha.C; E Mail: drhemac19@gmail.com

Received 23 ${ }^{\text {rd }}$ July 2021; Revised $27^{\text {th }}$ Aug. 2021; Accepted $30^{\text {th }}$ Sept. 2021; Available online $1^{\text {st }}$ Nov. 2021 https://doi.org/10.31032/IJBPAS/2021/10.11.1097

\section{ABSTRACT}

Technologies designed to deal with computationally intelligence have grown extremely efficiently, and in certain circumstances, they produce more accurate findings than humans judgments. As a result, this research presents a novel online strategy based on deep learnings techniques and the notion of transfers learning to develop a computational intelligence frameworks for use with IoHT devices. That frameworks makes it virtually as simple for users to upload photographs and do platforms education as it is to create folders and upload files to traditional hosting companies. This product's trials revealed that evens persons with no scripting or picture processing experience could build up applications in a matter of minutes. 3 medicals databases are used to validate the proposal method: pictures of cerebral 
vascular accidents for strokes type classifications, lungs nodules images for malignancy classifications, as well as skin pictures for melanocytes lesion segmentation. These findings indicate the application's performance as well as dependability, with 91.6 percent accuracy in the strokes plus lungs nodules datasets as well as 92 percent accuracy in the surface imaging collections. Our demonstrates the significant impact that this research may make in assisting medicals practitioners in swiftly as properly evaluating difficult tests, as well as enabling a vast medicals examination information to be accessed through an unified cooperative IoT Development kit.

\section{Keywords: Internet of Things; Image processing; Deep Neural Network; Innovations INTRODUCTION}

Advanced technologies have made it possible to create low-cost, user-accessible handheld computer systems. Cellphones having advanced in terms of technology as well as computing capacity, and therefore by connecting to mobile internet connections, smartphones became a resource, engagement, including exchange. Smartphones already have virtually grown vital in modern culture's day-to-day lives. According to studies, there are many more networked gadgets than humans on the earth as of 2008 , but this trend is expected to accelerate exponentially. Physically things are now being linked to the Internet thanks to technological innovations. This name "Internet of Things" (IoT) first meant to describe the usage of sensors as well as devices that could have been manipulated \& remotely accessed [1]. The IoT began to bridge the gaps between physiological devices and the Networks data, allowing for a much broader ranges of applications, not just for entertainments, as well as for the treatments and monitoring of healthcare needs, environmental surveillance, and connected home, among many other things.

Clouds technology, that gives computational powers and storages availability from every place with an Internet access, has been introduced to this situation. This expands the variety of resources accessible, dependent on the demand for a program for fairly modest fees and proportionate to do using, making such capabilities that much more approachable [2]. Multiple researches, and also the creation of software specialised for the health industry, such as healthcare management, were discovered during an experimental review of the literatures.This identification \& categorization of strokes is accomplished using an extraction of features 
approach called Assessment of Brains Tissue Thickness, which would be based upon brains radiological density patterns, in one of the research observed [3]. In [4] an intelligence IoT systems that delivers, analyses, and saves patients gait patterns data using an Inertial Measurements Units is offered to monitoring persons with diabetic, for instance, a condition that causes a drop in motor movements. A heart surveillance technology based on Raspberry Pi as well as IoT is being researched at [5]. Detector communications is handled using an Arduino-based microprocessor. The Raspberry $\mathrm{Pi}$ is used for all data processing. These outcomes of digital logic are delivered to the web application servers. [6] describes a systems that uses clouds and fog computing methods, as well as IoT\& Big Data technologies, to aid in the resolution of sleeping apnea-related restrictions.

\section{Related Works}

In [7], Deep Neurals Network is used to construct a cloud-based IoT solution to help in the medicals diagnosis of Chronic Kidneys Diseases. [8] proposes a real-time epileptic diagnosis systems that uses Discretes Wavelets Transforms, Hjorth Variables, Statistical Properties, \& Deep Neurals Network to interpret electroencephalography inputs. [9] also has a research that incorporates machines learning, big data, plus IoT. Even if they are standalone gadgets or systems that use devices to sense healths factors (hearts rate, oxygen, etc.), all data collected may be integrated into a single framework with the objective of making illness preventive as well as treatment easier.Also there are diagnostics help alternatives, most of which have been established by huge corporations, including such IBM's expensive Watson Healthcare system, as well as the usage of socials networking technologies for group \& patients monitoring [10]. Furthermore, commercial units for screening tests to allow remotes diagnosis are available.

In light of the present state of the arts, the present project distinguishes out because it combines picture processing with artificial intelligences to detect abnormalities as well as deliver diagnostics data in a setting that does not need a thorough understanding of the techniques involved. That work is particularly unique in that it incorporates an image recognition software that could be disseminated through such a social medialike platform, with the goal of increasing the utilisation of multiple datasets but also, as a result, increase the reliability of the techniques and methodologies utilised. It is indeed a significant steps in the present 
culturals framework of organizational learning, which is governed by cooperation \& allows experts to share their knowledges and expertise [11]. The approach combines multiple processors methodologies with the advantage of ensuring high accessibility including fostering straightforward usage by lay consumers of computing who already are unfamiliar with image processing techniques.

Such sort of software is a noteworthy barrier to create since it necessitates the integration of 3 aspects that are not often combined: Computers expertise, particularly in photo processing, clinical skills, as well as powerful computational capacity are all required. Numerous clinical diagnostic testing research programs may be discovered that employ different algorithms and also have highly exact results, however they lack an application that regular healths practitioners are using. Considering this, the suggested scheme aims to address these issues by providing a forum that can be used by anybody with a basic understanding of the basics sciences but still no particular expertise of diagnostics. As an users participates as well as distributes photographs on this network, the interfaces efficiency improves, as well as customers' works may be streamlined as a result.

\section{Proposed Method}

This team having product is called LINDA, that stands for Lapisco Imaging iNterface for such Developments of Applications, but it comprises of 2 components. Lapisco is the portuguese abbreviation for Laboratories of Image Recognition, Signaling, as well as Application Computation, and that it is the names of the developments group. First one, written in Java, provides a web services that handles data flow among the devices as well as the clouding platform. The module is interfaced to Postgre SQL, a free relationals database which records data about available extractors as well as classification techniques, pictures used only for model training, as well as a choice of separator and classification pairs which produce the best photo processing outcomes, and also processing official figures and outcomes. This also offers a user-friendly as well as responsive online application that enables customers make changes.

This devices secondary subsystems were written in Python as uses libraries like Tensor Flow \& Keras to analyze pictures received via Convolutional Neural Networks $(\mathrm{CNN})$. The usage among these frameworks assures that the techniques, as well as the information flows of the LINDA systems, are 
always implemented in the same manner. The predictions API would be available to use after the picture foundations have been established and the techniques have already been chosen. The picture will be sent from a machine to start the data flow. The picture to be analysed as well as the cryptographic hash values must then be sent to computer. This would cause the computer to contact the predictions API, that will choose the techniques to use depends on the security hashes. This framework setup would already have established appropriate techniques. That picture would then be adjusted to meet the handling criteria, allowing the systems to function with a variety of cams and image viewer methods without the need for extra user effort or modifications to equipment that are interconnected through web applications. These designs that are required would be put into storage.

Furthermore, whether the same modeling has already been requested lately, by even a various users or application, the modeling doesn't have to be rebuilt, resulting in a speed boost of up to $60 \%$ in certain circumstances. If indeed the program's configurations haven't been completed and modifications are necessary, the web apps path is utilised, that enables for picture uploads \& classification. This customer might construct so many more categories as he or she wants to categorise the photographs, for instance, 2 groups, one for normal lungs pictures as well as for nodules pictures. A thorough study of the photos necessitates at least two categories, each one with ten photographs. This same framework does have ten methodologies for attributes extraction as well as eight methodologies for categorization stages, that provides data such as correctness and time consumption in the form of graphs and charts after processing the registration images dataset, allowing the user can select the extractors includes classifier establish he requires. Originally, it must have been planned to make the best combination choice automated; but, in place to enable academics researchs and

scientifics investigations, it was chose to leaves the option to the consumer.

In the case of futures deployments, however, the customer would have the option of accepting the automated pick or making his / her own decision. As previously stated, the internet component were created in Java $\&$ utilises the Java Server Faces (JSF), Java Persistence API (JPA), \& Hibernate technologies. It is now accessible at http:/linda.lapisco.ifce.edu.br. This predictions API were created expressly to 
operate with both the planned systems, and also other systems \& technologies. As a result, in order to maintain excellent compatibility, the API can interact via JSON files, that are transmitted in a secured environment using the HTTPS protocols (port 443). XML documents were investigated throughout the developments process, however the JSON setups previously met the requirements, hence XML wasn't really incorporated. Futures versions might incorporate the usage of XML, allowing for evaluating performances. Nevertheless, because both a compression as well as a physically created document are delivered in a testing, either are almost the same sized, suggesting no difference in bandwidths use.

\section{Design Procedure}

At the time of its inception, the systems were separated into 4 components.
This 1st section, Resource-and-Events, is able to obtains pictures, whether it be for predictions or as a supplement, as well as the systems includes protection \& users interfaces aspects. This Context- and Analytics section is made up of the API predictions, it conducts characteristic extracts, picture categorization, and predictions, based on the situation. The section 3 is Persistent, that uses a Postgre SQL databases for storing the photos, systems setup information, as well as computational capabilities, but also associated outcomes.Interoperability's is the 4th and finals component, that allows systems integrations with other devices \& systems. All sections of the systems include security safeguards; nonetheless, the interoperable section has had no interaction.

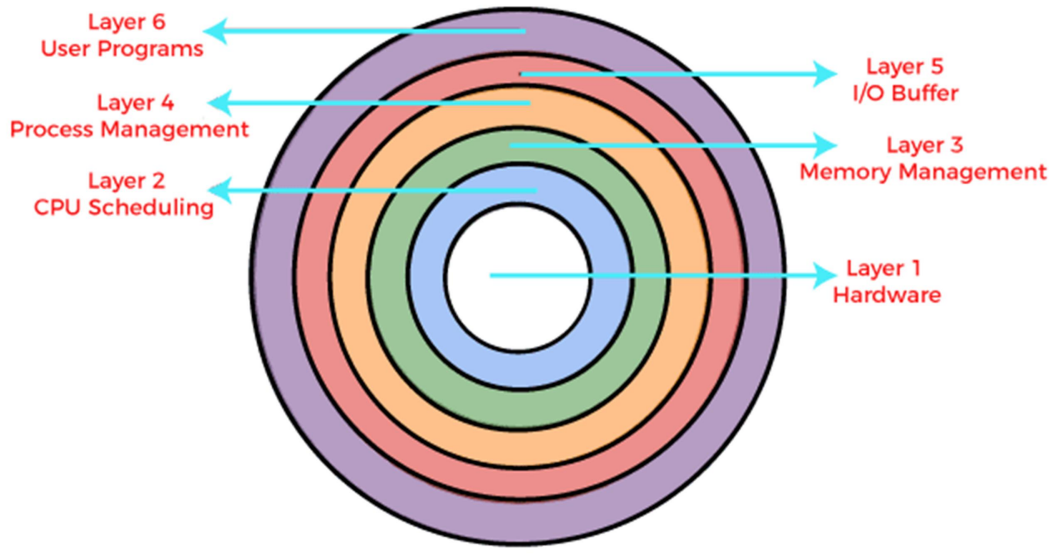

Figure 1: The layers of the LINDA system 


\section{System Requirements}

In additional to the LINDA system's security issues, that were regarded the key non - functional need throughout implementation, certain additional factors in this classification were evaluated, and that they are described following. Another of the program's aims was to ensure that the systems available web to anybody interested in fostering inquiry, therefore adaptability was a key feature. Because the web visualisation subsystems is completely detached from the photo processing section $\&$ performed out utilizing different technology, this seems to be achievable thanks to LINDA's modularized architecture. Information on computational resources utilisation would be supplied afterwards.Because of the kind of operations of LINDA as well as the demands of diverse research contexts, particularly for usage with clinical uses, it is critical to take care to keep the platforms accessible throughout all moments, specifically while upgrading. Once more, the program's modularity allows for the employment of multiple networks elements to ensure the availability of each of the required components. Inside the event of a complete failure of the image recognition subsystems, client requests are queued \& processed as soon as even the modules becomes accessible.In terms of the internet \& persistent, there seem to be numerous types of web application server redundancies that are straightforward to implement in this sort of solutions.

That technology which will be used for accessibility is unclear due to the variability of contexts including the Online; nevertheless, the usage of the online platforms already answers questions. Researching settings, on either hand, necessitate activities in conjunction with or related with other programs or technology, in addition to the Web.Externals systems having directly accessbility to databases or other network parts might lead to various coding errors as well as cybersecurity issues, this was one of the greatest considerations during in the system's implementation. As a result, a component with such a JSON files interchange formats and a hashed verification keys were created to facilitate interoperable. The objective was to make connecting to a large variety of present and futures platforms as easy as possible.

\section{Online Image Processing and Analysis}

Everyone's behaviour has changed dramatically as a result of technical advancements including increasing mobility of computing equipments. People's 
relationships have changed as a result of these developments. Nowadays, technologydriven interactions are quite widespread, with ICTs providing omnipresences that overcomes geographically and temporally limitations. That interdependence may be said to build virtuals communities, or virtuals communication networks that enables users to connect in a structured manner surrounding common goals, that are manifested in the form of devices \& materials shared by groups of individuals.Several literatures employ online platforms to address health issues, but they are dependent on the evaluation of patients as well as overall wellness through all these platforms. There were no papers that employed a specialised online community for data sharing, yet healthcare providers who knew each other might communicate their findings. Nevertheless, there was only direct contact between the participants for picture processing, with no cooperation thru a computer. In light of these factors, the LINDA systems was intended from the start to include an online community to encourage exchanges of knowledge including conversations about the outcomes of the activities generated inside the platform.

However there are others social networks including collaborations tools with a different emphasis, humans do not believe that they might impede the development of the developed framework so because aims of some of these technologies have quite a completely different targets audiences and objective than those envisaged in this project. Is from the other side, this would not rule out the potential of futures integration, but it'll be dependent on the demands that develop. As a result, the suggested applications will allows the program's base photos to be distributed across several customers; nevertheless, the originating users base is always preserved, while some others users could remove or add new pictures. Furthermore, the systems includes a cropped feature that enables consumers to restrict the number of picture spaces evaluated by the machine learning algorithms, allowing them to focused on a particular portion of an images without having to utilise a separate photo manipulation programme. Depends on the materials available in the created technique, no other research that integrated a quick and easy way to do computer vision with the ability to shares and adapt the produced understanding to the requirements of the each customer was identified in a survey of publications as well as magazines. If just the healthcare system is taken into account, the programmes are confined to team training including patients follow-up. 
Figure 2 depicts a high-level summary about how our strategy works. Firstly, professionals get photos from the Iot system then transfer them to the frameworks over the Web. Following that, LINDA performs a systems call to the predictions web application, delivering a hass code in conjunction to the picture given by the users. This Predictions API is then called, though it does picture preparation, that includes altering the picture's dimensions \&, if required, colour conversions but also bitrate, to guarantee correct functioning irrespective of the acquisition device utilized. These photos are therefore processed using $\mathrm{CNN}$ using machines learning techniques, as well as the diagnostic is sent to the professional, where it may be seen on a computers or mobiles, for instance.

The IoT Managements functions in the systems coordinates flow of data, regulates systems access, feels safe, and handles interaction with some other programs or equipment.

Spites of the progress in position, fragmentation, as well as object recognition offered by moderns CNN architectures, which were trialed and use very huge databases including such ILSVRC, COCO, as well as JFT, those same methods generally have 1000's of variables as well as, when tried to apply to visual information with very few measurements, would almost certainly result in overfits. As a result, using Transfers Learning, which has been employed in a number of studies, is an excellent technique to get around these restrictions. The primary principle behind transfers learning is to apply the "expertise" gained from one activity, including such item categorization, to a new one, such as detecting a strokes on Ct scanners. To apply transfers learning using $\mathrm{CNN}$, several stages must always be completed, including such developing and trainings a networks, including such VGG / ResNet networking using suitably large databases, comparable to ImageNet, and thereafter removing the levels nearest to the result. This leftover structure is then performed to every one of the photos in the database to be utilised, and each one of these is saved as a separate database, together with their annotations. This fresh information is then used to hire and train classification, including a Supports Vectors Machine / a Multilayer Perceptrons. 


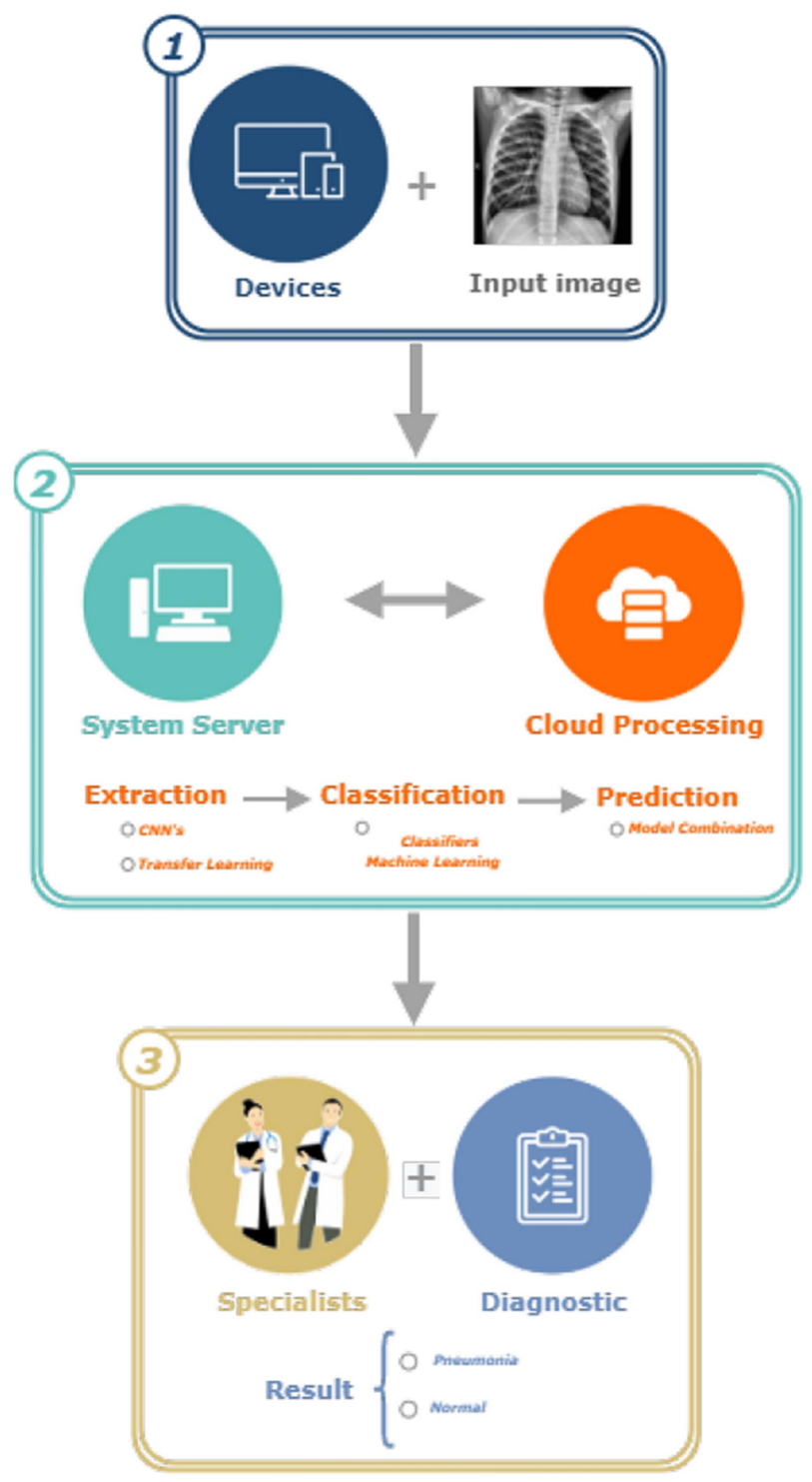

Figure 2: LINDA framework overview

\section{System test}

This systems was put through its paces for 2 months, with both the help of programs from our research group plus assistance from partners. The main objectives was to confirm that the original goal has been met, which would be to develop a basic framework that didn't requires extensive knowledge to use and to authenticate its assimilation with a networking site established by team, in such a way that what an user visits could works with the entire system without even any usability issues.Throughout this experiment, respondents answer a fivequestion assessment about ease of using it, whether the technology exceeded their objectives, whether they would suggest the 
technology to a friend, and whether the absence of information might make it difficult using the platform. Again from mean results provided to each of the inquiries, each with proportional allocation, the outcome was an averaged favourable review of 87 percent.

On 25 computerised CT scans pictures of cerebral vascular accidents (CVA), commonly known as brains strokes, the very first controlled as well as achievement tests were performed. There must have been 420 CT pictures in the dataset, 140 of normal skulls as well as 280 of skulls with strokes, 140 of hemorrhagic strokes and 140 of ischemic strokes. As seen in the diagram, the information is separated into three categories. This PH2 Collection, a dermoscopic picture collection for study \& comparison, was used for the $3 \mathrm{rd}$ supervised experiment. The photos in this collection are obtained from the Clinic Pedro Hispano's Dermatological Services (Matosinhos, Portugal). Over all, there are 120 dermoscopy images pictures of melanocytics diseases in the databases, including 80 examples of ordinary nevi as well as 40 specimens of melanomas. That information is open to the general public and can be downloaded from the webpage [12].

RESULTS AND DISCUSSION
This Gaussian probability distribution functions was used by the Bayesian classifiers in the categorization procedures. An grids search was also used to choose the hyper - parameters $\mathrm{k}$ from of the $\mathrm{kNN}$ classifiers, trying odd numbers from 1 to 11. 3000 predictions were employed in the Randomized Forests training procedure. MLP trained to use the Levenberg-Marquardt technique in the hidden units, with neurons ranging from 2 to 1000 . The LINDA architecture runs all possible extraction \& classification pairs as well as chooses the appropriate outcome for every application. The extraction plus classification combinations that makes the greatest outcome for a given data set, based on accuracy \& F1-Score in this manner.If such two factors were similar, the overall duration, which is the summation of the training and validation periods, is next criteria to evaluate. While the $\mathrm{kNN}$ as well as SVM (RBF) classifications are employed in combination with the all the CNN architectures for the CVA databases, the method achieves 100\% reliability with F1-measure. Bayes with VGG16, VGG19, MobileNet, InceptionV3, Xception, InceptionResNetV2, DenseNet121, DenseNet201, DenseNet169, as well as NASNet Mobile, as well as $\mathrm{kNN}$ using VGG16, VGG19, as well as DenseNet169, 
had the quickest trainings schedules of $0.1 \mathrm{~s}$. Bayes' categorization speeds were likewise the quickest among all CNN designs, requiring only 0.2 seconds.

Research findings for lungs nodule categorization revealed that SMV (RFB) as well as MLP in conjunction with Inceptions V3 \& Exception, correspondingly, had a reliability of 89.1 percent. Mixing SVM (RFB) with Inceptions V3 resulted in the greatest F1-Score result of $85.8 \%$. Whenever it came to Exception, SVM (RFB) \& SVM (Straight) earned 85.5 percent. Bayes produced the best categorization as well as testing times in conjunction with all
CNN designs, which have been less than 0.1s.DenseNet201 in conjunction with $\mathrm{k}$ Nearest Neighbors produced 94.0 percent accuracy \& 93.2 percent F1-Score inside the skins dataset. Whenever MobileNet was paired with any of the systems classifications, it achieved 90.0 percent accuracy, as well as the SVM (Regular) earned and over 88.0 percent for the F1Score whether integrated using DenseNet121, DenseNet169, or VGG16. Whenever MobileNet were paired using Bayes, the quickest waiting period has been less than 0.01s. In Table 1, the top findings for every dataset are shown.

Table 1: Proposed system test

\begin{tabular}{|c|c|c|c|}
\hline Dataset & Classifier & Accuracy in \% & Time to process (s) \\
\hline CVA & K-Means & 100 & 1.6 \\
\hline LUNG & SVM & 91 & 2 \\
\hline SKIN & K-neighbour & 95 & 2.4 \\
\hline
\end{tabular}

Its combination of a medicals image processing system with such an online community is one of the work's primary distinguishing features. Our goal of this connection is to enhance the accuracy of the findings by collaborating with other information systems who enable the usage of respective information. Information must always be made accessible in a sharing of resources platform. For assess the benefits of cooperation, the developments staff was separated into 3 teams, within each developments area including responsibility for photo editing based on the information gathered. The goal here was to not enhance precision, but seeing how more cooperation might result in better outcomes.Each AVC basis was divided into 3 areas in first testing, with 2 (2) groups receiving 47 samples out of each basis and just one (single) receiving 46 samples out of each. The accuracy rates achieved when the operations are performed 
out separately were 81.2 percent, $87.7 \%$, and $78.9 \%$, respectively. The reliability climbed to 93.6 percent when the two groups to the most samples pooled their foundations, which would have been a gain of around 15.27 percent over the poorest outcome; nevertheless, the processed time will increased. Variances in computation are deemed less important than correctness, particularly when certain variability do not exceed one sec (1s)

This pulmonary nodules foundation the previous experiment, splitting the group into 3 (three) groups. Independent accuracy outcomes are 65.2 percent, 73.4 percent, as well as 69.9 percent, respectively. When the suggested solutions foundations were shared, the biggest gains of merging 2 of the two levels climbed to 80.1 percent, representing a 22.85 percent increase in accuracy through the worst outcome. Figure 3 shows the findings of the lung nodules and AVC datasets.

was then tested again, using same methods as

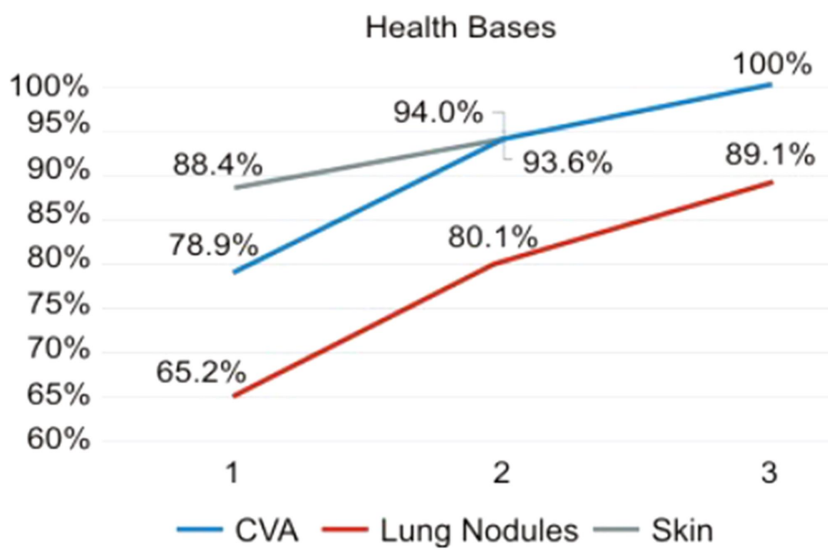

Figure 3: Comparison of the results of collaboration

Melanoma was the most recent test done using the health-related foundations, however due to the lesser sample size, it was only divided into 2 (two) portions rather than 3 (three). The reliability findings for separate processes were 88.4 percent and 90.1 percent, respectively, with an accuracy improvement of 6.33 percent when employing the entire base, bringing the total to 94.0 percent. Such findings demonstrate the value of cooperative process, benefiting everyone those that use the solutions for diagnostics, by gaining more consistent readings, and those who works with image analysis, by allowing them to test innovative techniques on bigger research bases.

\section{CONCLUSIONS}

This construction of a cooperative online community to help in diagnosis of diseases was suggested in this study. The 
objective was to create machines learning technologies easier to use even for those who understand about them, and to also help advance for peoples who don't know anything about cognitive computing, arithmetical having to learn, or convolutional neurals network (cnn to include them in a structured and concise manner. When these techniques are combined with a structure that maximises cooperative possibilities, they may produce a substantial body of knowledge for each challenge and encourage positive interdependence. There at conclusion of the project, the application provides a substantial benefit by allowing customers to do a working prototype utilising deep learning and other sophisticated computerbased approaches while also including their datasets and setups. Individuals may also share their information with other colleagues and social media networks members. As a result, the consumers have validated their assumption and then have a suite of products at the conclusion of the procedure. This program's accessibility was highly rated by 87 percent of the consumers that answered to the survey.Additionally, the combination of the online community with the programme, that employs deep machines learning ideas, allows for the cooperative as well as centralised use of artificial intelligences technologies. Samples are included in the, and the generated application is utilised collaboratively in contrast to permitting the changeover of diverse users in a program. Furthermore, the reliability of the findings was strengthened, although had already been excellent with the basic usage of existing techniques. Because when picture collections were expanded thru cooperation supplied by the online community, those who increased by even more that $22 \%$. It is indeed worth noting this, in additional to the health picture datasets mentioned above, the programme has been used on various sorts of picture datasets, including manufacturing applications including equipments evaluation. The outcomes were likewise strong, with reliability increases of over $15 \%$ achieved using the instrument, but they have been not reported in further depth and they were not the subject of this study. Researchers do, nevertheless, emphasise the advantages of someone using digital platforms in this situation.

\section{REFERENCES}

[1] Parvathy VS, Pothiraj S, Sampson J. Optimal Deep Neural Network model based multimodality fused medical image classification. Physical Communication. $2020 \quad$ Aug 1;41:101119. 
[2] Boveiri HR, Khayami R, Javidan R, Mehdizadeh A. Medical image registration using deep neural networks: A comprehensive review. Computers \& Electrical Engineering. 2020 Oct 1;87:106767.

[3] Ashraf R, Habib MA, Akram M, Latif MA, Malik MS, Awais M, Dar SH, Mahmood T, Yasir M, Abbas Z. Deep convolution neural network for big data medical image classification. IEEE Access. 2020 Jun 1;8:10565970.

[4] Cai L, Gao J, Zhao D. A review of the application of deep learning in medical image classification and segmentation. Annals of translational medicine. 2020 Jun;8(11).

[5] Yao X, Wang X, Wang SH, Zhang YD. A comprehensive survey on convolutional neural network in medical image analysis. Multimedia Tools and Applications. 2020 Aug 24:1-45.

[6] Gupta V, Demirer M, Bigelow M, Little KJ, Candemir S, Prevedello LM, White RD, O'Donnell TP, Wels M, Erdal BS. Performance of a deep neural network algorithm based on a small medical image dataset: Incremental impact of 3D-to-2D reformation combined with novel data augmentation, photometric conversion, or transfer learning. Journal of digital imaging. 2020 Apr;33(2):431-8.

[7] Kalinin AA, Iglovikov VI, Rakhlin A, Shvets AA. Medical image segmentation using deep neural networks with pre-trained encoders. Deep Learning Applications. 2020 Feb 28:39-52.

[8] An FP, Liu JE. Medical image segmentation algorithm based on optimized convolutional neural network-adaptive dropout depth calculation. Complexity. 2020 May 15;2020.

[9] An FP, Liu JE. Medical image segmentation algorithm based on optimized convolutional neural network-adaptive dropout depth calculation. Complexity. 2020 May 15;2020.

[10] Thurnhofer-Hemsi K, Lopez-Rubio E, Roe-Vellve N, Molina-Cabello MA. Multiobjective optimization of deep neural networks with combinations of Lp-norm cost functions for 3D medical image super-resolution. Integrated 
Computer-Aided Engineering. 2020 Jan 1;27(3):233-51.

[11] Hassanzadeh T, Essam D, Sarker R. EvoU-Net: An evolutionary deep fully convolutional neural network for medical image segmentation. In Proceedings of the 35th Annual ACM Symposium on Applied Computing 2020 Mar 30 (pp. 181189).
[12] Bhattacharya S, Maddikunta PK, Pham QV, Gadekallu TR, Chowdhary CL, Alazab M, Piran MJ. Deep learning and medical image processing for coronavirus (COVID-19) pandemic: A survey. Sustainable cities and society. 2021 Feb 1;65:102589. 